\title{
Comparison of Bit Error Rate of Line Codes in NG-PON2
}

\author{
Tomas Horvath, Radek Fujdiak, Milan Cucka, Marie Dankova and Jiri Misurec
}

\begin{abstract}
This article focuses on simulation and comparison of line codes NRZ (Non Return to Zero), RZ (Return to Zero) and Miller's code for NG-PON2 (Next-Generation Passive Optical Network Stage 2) using. Our article provides solutions with Q-factor, BER (Bit Error Rate), and bandwidth comparison. Line codes are the most important part of communication over the optical fibre. The main role of these codes is digital signal representation. NG-PON2 networks use optical fibres for communication that is the reason why OptSim v5.2 is used for simulation.
\end{abstract}

Keywords-Miller's code, RZ, NRZ, NG-PON2, Matlab, Simulations

\section{INTRODUCTION}

The first standard above 1 Gbps was GPON. GPON standard was improved by ITU-T in 2003. The following standard was XG-PON, which has $10 \mathrm{Gbps}$ bandwidth. In general, the transition from GPON to XG-PON had a disadvantage because the ISP needed to change PIN photodetector onto APD photodetector in ONU unit. All mentioned standards came from ITU-T. The second group, which has provided recommendation for passive optical networks, is an IEEE group. In general, standards of IEEE group are based on Ethernet technology. That is the reason why these standards dominate in Asian access networks. The latest standard NGPON2 still covered by commonly line codes NRZ and RZ define in general requirements. Miller's code is mentioned only in appendix such as next possibility.

This paper deals with line codes in NG-PON2 standards for passive optical networks. On the other hand, nowadays only general requirements have been done [1]. In general, access networks have used the same line codes for a long time (from GPON to NG-PON2). In [2], Miller's code was mentioned such as the new possibility for using in the new optical networks.

This article presents a new way of using Miller's code in access networks for the latest standard. Miller's code is known as a decoder for delay-modulation coded Data [3]. The mentioned article was published in 1971. Nowadays, line codes are still from OOK (OnOff Keying) which are not the most bandwidth efficient. We designed Miller's coder in Matlab and implemented it into OptSim simulation application due to the comparison line codes: NRZ, RZ and Miller's coded in NG-PON networks.

Research described in this paper was financed by the National Sustainability Program under grant LO1401. For the research, infrastructure of the SIX Center was used.

Manuscript received April 12; 2016 revised May 20, 2016.
The main contribution of this paper is the determination of the maximum bit error rate value in the transfer of 3 different line codes in NG-PON2 networks. This is followed by the research into effects of increasing spectral width of laser on bit error rate with different line codes.

The rest of this paper is structured as follows. Section 2 gives an overview of some other related works. Section 3 describes the line codes in our model. Section 4 shows the designed model and parameters of blocks. Section 5 discusses the results achieved and Section 6 concludes the paper.

\section{STATE OF ART}

Many works related to a comparison of the line codes have been published in recent years. Works published these days are aimed at a comparison of line codes presented in simulation applications as a NRZ, RZ, etc.

Authors in [4] investigates the suitabillity of various data formats for optical XOR gate at $10 \mathrm{~Gb} / \mathrm{s}$. They focuse on different data formats for high-speed optical networks in XOR gate. NRZ, RZ, and Manchester modulation format was tested and verified. On the other hand, they used only simple line codes, where Miller's code is missing. In NG-PON2 networks the optical amplifiers have not been presented in distribution network. In the next publication [5] the influence of binary modulations was presented. They used NRZ, RZ, CSRZ, and CSRZ line codes for their research. On the other hand, Miller's code was not included. Further, the publication [5] used only 2.5 Gbps in simulation model. Our model has $40 \mathrm{Gbps}$ which is a combination with four $10 \mathrm{Gbps}$ transmission system.

Other articles [6], [7], deal with the same line codes as was mentioned above. None of them contain Miller's code, known as Sigma-Delta modulation, the articles compared only the base line codes, which are proposed in simulation networks. In general, Miller's code is able to improve a bit error rate in coexistence scheme and single technology network. The last article [8] deals with PMD (Polarization Mode Dispersion) for NG-PON, more precisely 10 Gbps network.

The main proposed reason of our article is the comparison of the current line codes with the known Miller's code from data backup field. Another one [9] deal with coexistency the three latest standards. On the other hand, the paper does not deal with influence of Miller's code in coexistence network.

Our work focuses on a design and implementation of well-known code for visible light communication [10], called Miller's code. The main contribution of this paper is a comparison of the included line codes with our implementation of Miller's code in OptSim v5.2 Miller's code brings better results for attenuation values. 


\section{NG-PON2}

We dealt only with the latest standard ITU-T G.989 called G-PON2. A general specification is available in [11]. This standard was improved in March 2013. First, the study group 15 had the main goal to select the main technology for these networks. The study group 15 had many technologies for their decision: WDM-PON (Wavelenght Division Multiplexing PON) [12], OFDM-PON (Orthogonal Frequency Division Multiplexing PON) [13], UDWDM-PON (Ultra Dense WDM PON) and TWDM-PON (Time and Wavelenght Division Multiplexing PON) [1]. In the final version TWDM-PON technologies have been selected. TWDM technology needs to be changed only at ONU side that means ISP (Internet Services Provider) should change ONU units, due to TWDM technology required the tuneable laser at customer side. On the other hand, there is no change in attenuation classes for passive optical networks according to [1] or [2], see in Tab. I.

TABLE I

ATTENUATION CLASSES FOR NG-PON2

\begin{tabular}{|c|c|c|c|c|}
\hline & Nominal1 & Nominal2 & Extended1 & Extended2 \\
\hline Minimum loss $[\mathrm{dB}]$ & 14 & 16 & 18 & 20 \\
\hline Maximum loss $[\mathrm{dB}]$ & 29 & 31 & 33 & 35 \\
\hline
\end{tabular}

In general, TWDM-PON networks are the combination of four or eight channels, which are the same as in XG-PON but they use wavelength of NG-PON2 spectrum (see in Tab. II).

TABLE II

WAVELENGTHS FOR DOWNSTREAM COMMUNICATION (OLT TO ONU)

\begin{tabular}{|c|c|c|}
\hline \multicolumn{3}{|c|}{ Downstream } \\
\hline Channel & $\mathrm{f}[\mathrm{THz}]$ & $\lambda[\mathrm{nm}]$ \\
\hline 1 & 187.8 & 1596.3389 \\
\hline 2 & 187.7 & 1597.1894 \\
\hline 3 & 187.6 & 1598.0408 \\
\hline 4 & 187.5 & 1598.8931 \\
\hline 5 & 187.4 & 1599.7463 \\
\hline 6 & 187.3 & 1600.6004 \\
\hline 7 & 187.2 & 1601.4554 \\
\hline 8 & 187.1 & 1602.3113 \\
\hline
\end{tabular}

\section{LINE CODE}

Line codes also called digital baseband modulation is used for telecommunication systems. It is an amplitude and timediscrete signal which is specially tuned for the channel. Line coding is made from a signal which is represented by voltage and in digital expression zeros and ones. If a sender and a recipient communicates, this process is called coding lines. There are several types of encoding: polar, unipolar, bipolar, and Manchester encoding.

Selecting the appropriate code in the system design is an important step. The same encoding cannot be used for copper and fibre optic lines because optical lines cannot have negative values. This problem is solved by the permanent preload, tristate code or unipolar code. The next option is transfer from bipolar signal to binary form and transfer to suitable line code.

The line signal is given by several requirements, such as high levels of clock components, constant DC component, minimum bandwidth, simple error detection etc. Optical transmission has several requirements: the performance and linearity modulation characteristics of the radiation source, attenuation and bandwidth cable or noise characteristics of the detector. In coding with line code NRZ and RZ codes are commonly used for their simplicity. Long sequences of ones and zeros are excluded by a scrambler.

\section{A. Return to zero - $R Z$}

Encoding processes of zeros and ones use positive and negative pulses. It is important to return to the neutral state. This means that there is zero voltage. The voltage is used to synchronize the clocks of the sender and recipient and all without the use of a clock signal. For coding greater bandwidth is required.

The binary signal is encoded by rectangular pulse amplitude modulation with this type of code. In coding return to zero inverted (RZI) is also used. It is a method of mapping for transmission. There are two levels of RZI signal. The signal has a pulse if the binary signal is zero and if the binary signal has no pulse, it is one. This method uses serial infrared IrDA physical layer specification.

\section{B. Non return to zero - NRZ}

In this coding logical ones mean specific value and logical zeros mean another specific value. This can be a positive or negative voltage. The signal contains no other values. Non return to zero encoding cannot be used for synchronizing transmissions. If there is a need to synchronous transfer, it must add synchronization with RLL (Run Length Limited) or add clock signal.

WDM also called Wavelength division multiplexing is used for combining multiple signals on laser beams at various infrared wavelengths for transmission along fibre. Laser generates a different type of signals. Laser is modulated by independent set of signals. Wavelength sensitive filters are used at the receiving end.

WDM modulation is similar to FDM which uses radio frequencies, but in WDM IR portion of the electromagnetic spectrum is used.

In a WDM system, a signal in each channel has a different carrier frequency, and the model equation of the system can be obtained by setting the electric field $q$ of the NLS equation [14]:

$$
i \frac{\partial q}{\partial Z}+\frac{\beta_{2}}{2} \frac{\partial^{2} q}{\partial T^{2}}+v|q|^{2} q=0
$$

in term of:

$$
q=\sum_{j=1}^{n} q_{j}
$$

where $q_{j}$ represents the electric field in the $\mathrm{j}$-th channel having carrier frequency, and $\omega_{j}$ means two channels,

$$
\begin{aligned}
& i \frac{\partial q_{1}}{\partial Z}-\frac{\beta_{2}}{2} \frac{\partial^{2} q_{1}}{\partial T^{2}}+\left(\left|q_{1}\right|^{2}+\alpha\left|q_{2}\right|^{2}\right) q_{1}=0, \\
& i \frac{\partial q_{2}}{\partial Z}-\frac{\beta_{2}}{2} \frac{\partial^{2} q_{2}}{\partial T^{2}}+\left(\left|q_{2}\right|^{2}+\alpha\left|q_{1}\right|^{2}\right) q_{2}=0,
\end{aligned}
$$


four channels, $\alpha=1$ (four channels are used in NG-PON2 topology with combining four $10 \mathrm{Gbit}$ data source),

$$
\begin{aligned}
& i \frac{\partial q_{1}}{\partial Z}-\frac{\beta_{2}}{2} \frac{\partial^{2} q_{1}}{\partial T^{2}}+\left(\left|q_{1}\right|^{2}+\left|q_{2}\right|^{2}+\left|q_{3}\right|^{2}+\left|q_{4}\right|^{2}\right) q_{1}=0 \\
& i \frac{\partial q_{2}}{\partial Z}-\frac{\beta_{2}}{2} \frac{\partial^{2} q_{2}}{\partial T^{2}}+\left(\left|q_{1}\right|^{2}+\left|q_{2}\right|^{2}+\left|q_{3}\right|^{2}+\left|q_{4}\right|^{2}\right) q_{2}=0 \\
& i \frac{\partial q_{3}}{\partial Z}-\frac{\beta_{2}}{2} \frac{\partial^{2} q_{3}}{\partial T^{2}}+\left(\left|q_{1}\right|^{2}+\left|q_{2}\right|^{2}+\left|q_{3}\right|^{2}+\left|q_{4}\right|^{2}\right) q_{3}=0 \\
& i \frac{\partial q_{4}}{\partial Z}-\frac{\beta_{2}}{2} \frac{\partial^{2} q_{4}}{\partial T^{2}}+\left(\left|q_{1}\right|^{2}+\left|q_{2}\right|^{2}+\left|q_{3}\right|^{2}+\left|q_{4}\right|^{2}\right) q_{4}=0 .
\end{aligned}
$$

\section{Miller's code}

Miller's code is used for Pseudorandom Binary Sequence (PBS). This code is known as delay modulation or modified frequency modulation. It contains rectangular pulses with two levels. Binary zeros cause no change of signal level. If this zero is followed by another zero, it means that transition to the other level takes place at the end of the first bit period. Binary ones mean transition from one level to the other in the middle of the bit period. Miller's code is primarily used for encoding radio signals and data backup. Frequency spectrum of the encoded signal contains less low-frequency energy than a non-return to zero signal and less high frequency energy than a biphase signal.

$$
\begin{aligned}
& S(\omega)=\frac{2}{\omega^{2} T[17+8 \cos 4 \omega T] \cdot\left[23-2 \cos \frac{\omega T}{2}-22 \cos \omega T\right]} \cdot \ldots \\
& \ldots \cdot\left[-12 \cos \frac{3 \omega T}{2}+5 \cos 2 \omega T+12 \cos \frac{5 \omega T}{2}\right]+\ldots \\
& \ldots+\left[2 \cos \frac{3 \omega T}{2}-8 \cos \frac{7 \omega T}{2}+2 \cos \frac{4 \omega T}{2}\right]^{2}
\end{aligned}
$$

\section{OPTSIM}

The simulation software has been developed by Synopsis. The main advantage of this application is a real time simulation of the real networks. Of course, all parameters are described via math equations which mean that some results are idealized. The application is able to simulate the following optical technologies: CWDM, DWDM, OTDM, FTTx etc. On the other hand, we can simulate analog signals of various modulation format too. The most important property of OptSim is cooperation with external applications Matlab and Spice, for example. Our solution of Miller's code was completely designed in Matlab.

\section{Simulation MODEL}

At first, we needed to design and implement Miller's code into OptSim application. Miller's code method (Delay method) is not implemented only in OptSim but also in other simulation tools. Our used simulation tool OptSim allows implementation and developing only Matlab environment possibility as functional matlab block. Matlab block in OptSim could have only electrical and optical input and output. It means for us that it is also not possible to use the logical sources (generators etc.), which are used for example with RZ or NRZ functional blocks. Miller's code, which is used for our simulation, was implemented in Matlab code. Our code provides also functional random generator, which is able to generate bits in necessarily speed for the system.

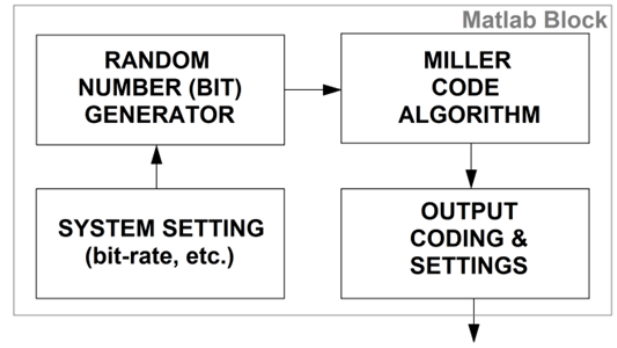

Fig. 1. Matlab code schematic

Matlab code schematic can be seen in Fig.1. The Matlab block has not inputs, because it was not possible to use logical sources for it and we create our source for the system. System settings include initiation data, bit-rate, a number of bit settings and also all other necessarily inputs and settings for our random generator and the whole system. The generator creates random bit sequences, which are coded by Miller's code algorithm. The output coding and settings only change Miller's code sequences to the right format for the OptSim simulator and it is also possible to choose there, for example, amplitude of the signal and some smaller and less important settings.

$$
\mid \begin{aligned}
& \mathbf{I F}(\mathbf{k}=\mathbf{0})=>\text { first type of logical one; } k=1 \\
& \mathbf{I F}(\mathbf{k}=\mathbf{1})=>\text { secondary type of logical one; } k=0
\end{aligned}
$$

Fig. 2. Main principal of coding

Miller's code has two types of logical one, it is necessary to hold some control variable, which will give order to a final sequence. The simple explanation of algorithm is in Fig.2. The generator generates bits, which goes to Miller's code algorithm, where the Miller-code sequence is created. First, each bit $(1$ or 0$)$ is extended by time and speed variable $(10 \mathrm{Gbps})$ and after it is with the Miller code algorithm (Fig.2) changed the logical 1 to the Miller logical one (to the first or second type). The technology NG-PON2 voids from combination four (40 Gbps) or ( $80 \mathrm{Gbps}$ ) sources based on ITU-T G.987. That means the wavelength multiplexer and demultiplexer must be included in real network and in our simulation model. We selected four $10 \mathrm{Gbps}$ data source wavelength of NGPON2 range, in concrete 1596.3389, 1597.1894, 1598.0408, and $1598.8931 \mathrm{~nm}$.

For the NG-PON2 networks four attenuation plans in two groups were proposed: nominal1, nominal2, extended1, and extended 2 (see Tab. 1.) For example, extended 1 and extended 2 attenuation classes required APD diode in ONU unit. On the other hand, we designed model only with APD diodes due to reflecting a recommendation. Our simulation model provides the total attenuation of $34.5 \mathrm{~dB}$ (in the last scenarios).

NG-PON2 networks have four parts. Each transmitting part contains the following properties: $10 \mathrm{Gbps}$ bitrate with pseudorandom sequence, wavelength of NG-PON2 range, various line code, $\mathrm{CW}$ laser, and modulator. Topology can be seen in Fig. 3. 


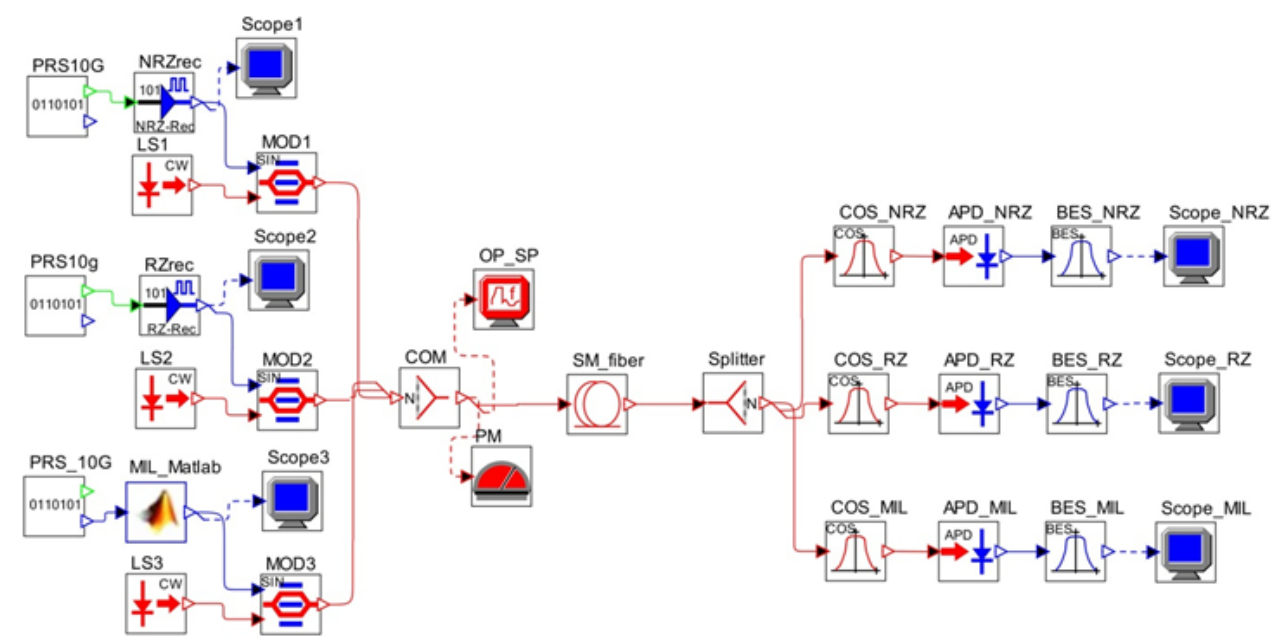

Fig. 3. Proposed topology for simulation with various line codes

As obvious in Fig. 3. we designed three optical sources with different line codes. First, we used NRZ line code, the second one was RZ. Both of them were carried out by Synopsis application OptSim. The third one for a comparison we need to implement Miller's code via Matlab.

Optical distribution network contained WDM combiner, single mode optical fibre with $20 \mathrm{~km}$ length, and splitter with different split ratio. The main goal of our article is the comparison of well-known line codes, which are used in the optical networks with Miller's code that were used at first in [3].

We created 2 scenarios with 8 values of split ratio (from 1:2 to 1:256 and the higher attenuation value) and 2 higher values than it was defined in E1 attenuation class (28 and $29.5 \mathrm{~dB}$ ). The selected attenuation values could be seen in Tab. III.

TABLE III

THE ATTENUATION VALUES FOR SPLITTER IN ODN

\begin{tabular}{|c|c|}
\hline Split ratio & Attenuation $[\mathrm{dB}]$ \\
\hline $1: 2$ & 3.6 \\
\hline $1: 4$ & 7 \\
\hline $1: 8$ & 9.6 \\
\hline $1: 16$ & 13.2 \\
\hline $1: 32$ & 17.4 \\
\hline $1: 64$ & 20.3 \\
\hline $1: 128$ & 23.5 \\
\hline $1: 256$ & 27.1 \\
\hline
\end{tabular}

Above mentioned merits are only for splitter, we need to add another attenuation of the rest component for total attenuation. In the final scenario we had $1+5+27.1=33.1 \mathrm{~dB}$. On the other hand, the recommendation ITU-T defined $35 \mathrm{~dB}$ as the maximum values. Due to a standard definition of attenuation maximum value, we had the last scenario with total attenuation of $35.5 \mathrm{~dB}$ in the optical distribution network.

\section{Simulation RESUlTS DISCUSSION}

All results are discussed with achieved BER values. As was already mentioned, the main parameter of our simulation is bit error rate. Another parameter is Q-factor, which describes the analog quality of digital signal with respect to SNR (Signal to Noise Ratio). Q-factor is defined by the following equation:

$$
Q=\frac{I_{1}-I_{0}}{\sigma_{1}+\sigma_{0}}[-],
$$

where $I_{1}$ logic level $1, I_{0}$ logic level $0, \sigma_{1}$ standard deviation of the logic level $1, \sigma_{0}$ standard deviation of the logic level 0 [3].

When we know Q-factor, we can define BER with the following equation:

$$
B E R=\frac{1}{2} \operatorname{erfc}\left(\frac{Q}{\sqrt{2}}\right) \approx \frac{\exp \left(\frac{-Q^{2}}{2}\right)}{Q \sqrt{2}}[-] .
$$

First of all, we designed a scenario for simulation of the maximum attenuation in ODN (Optical Distribution Networks) and we examined the values of bit error rate. As was mentioned in the previous chapter, final attenuation was $35.5 \mathrm{~dB}$ for our simulation model. The results of BER took a logarithm and the values were depicted in graph, which can be seen in Fig. 4

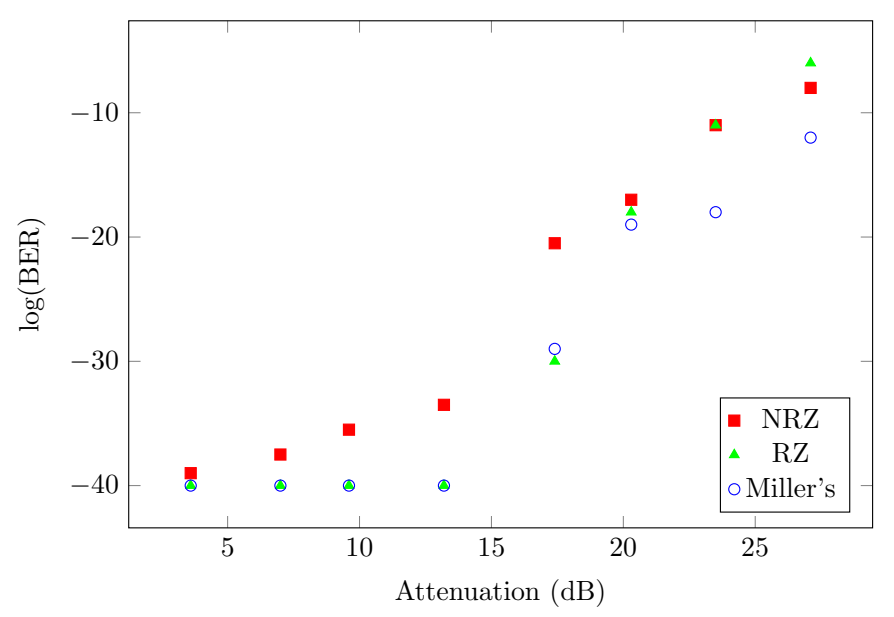

Fig. 4. Dependence of bit error rate on the attenuation value 
In Fig. 4 it can be seen that the Miller's code has one of the best results for attenuation values. In particular, Miller's code had BER $10^{12}$ for $27.1 \mathrm{~dB}$, NRZ had $10^{7}$, and RZ had $10^{6}$. In comparison, according to [1] only Miller's code should be accepted as applicably value. Source [1] defined the border BER value as $10^{12}$, which should be accepted in NG-PON2 networks. As was mentioned above, NRZ and RZ line codes had these values for the lower split ratio 1:128 $(23.5 \mathrm{~dB})$. We did not implement the FEC (Forward Error Correction).

The comparison of the eye diagram for split ratio 1:64 for Miller's and NRZ line code can be seen in Fig.5 Miller's code is represented in red and NRZ is in blue. From this eye diagram the following parameters should be compared: among others jitter and decision point. The values of jitter are $0.02 \mathrm{~ns}$ for NRZ and half of them $0.01 \mathrm{~ns}$ for Miller's code. In general, these values are completely sufficient because VoIP (Voice over Internet Protocol) required maximum jitter value of $150 \mathrm{~ms}$. Another highlighted parameter is a decision point. In comparison with NRZ it has better value that Miller's code, but the eye diagram of NRZ is not so clear as Miller's code.

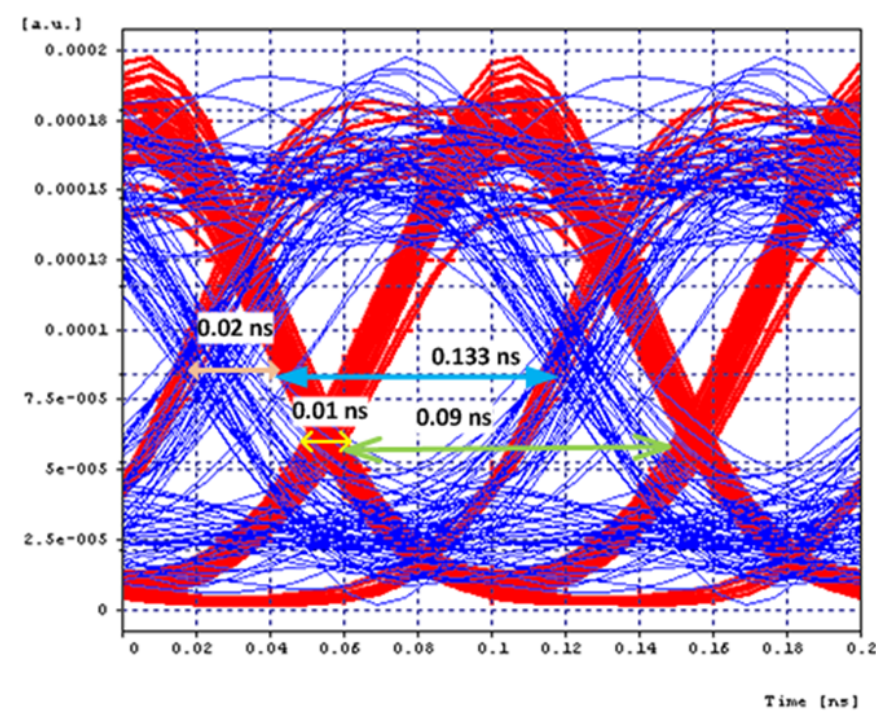

Fig. 5. The comparison of Miller's and NRZ eye diagram

Our scenario was divided into two parts, the results of the first part are described above. The second part of the scenario discovered the influence of FWHM on bit error rate. In simulation, the spectral width of the laser with $10 \mathrm{MHz}$ (default value) step was increased to $100 \mathrm{MHz}$. The aim was to achieve, as in the previous simulation, the boundary values of bit error rate, BER. On the average our designed Miller's code has one of the best results for this simulation. For example, $\mathrm{NRZ}$ and RZ had from $30 \mathrm{MHz}$ to $50 \mathrm{MHz}$ linear character for another increasing spectral width BER rapidly dropped on the border value. The overall decline was 3 orders in bit error rate. The resulting graph of the simulation is shown in Fig. 5 Simulated topology was still the same as the previous one but for this scenario we used split ratio 1:128, optical power of laser was $6 \mathrm{dBm}$ for all channel, and $20 \mathrm{~km}$ of optical fibre length. For the simulation topology must be the same, because of spectral with and their effect. If we have maximal split ratio, the results are incomparable and misguided. The next step was determination of the maximum bit error rate value in the transfer of three different line codes in NG-PON2 networks. This is followed by the research into effects of increasing spectral width of laser on bit error rate with different line codes Fig.6

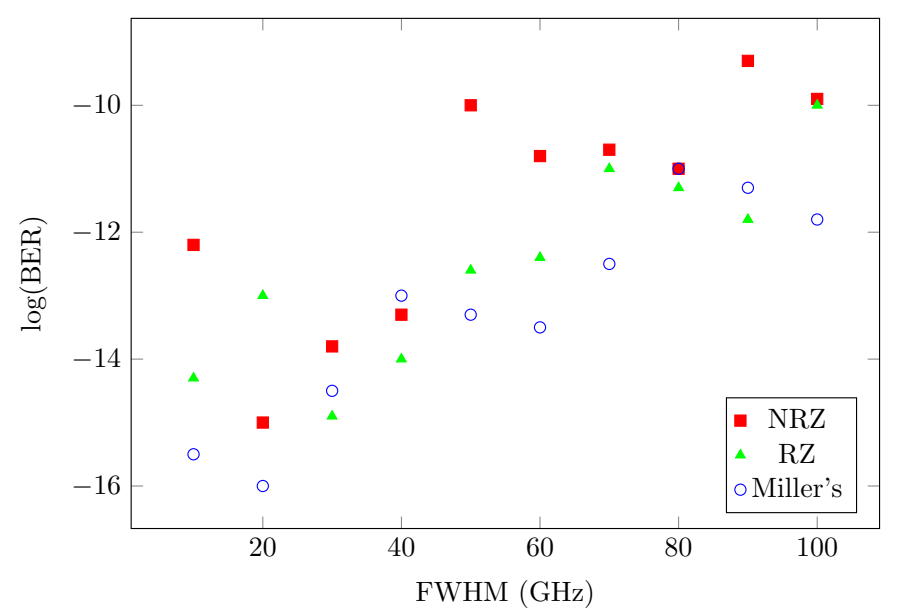

Fig. 6. Dependence of bit error rate on the spectral width of the laser

\section{CONCLUSION}

This article proposed a comparison of line codes with our implementation of Miller's code in OptSim. The next step was determination of the maximum bit error rate value in the transfer of three different line codes in NG-PON2 networks. This is followed by the research into effects of increasing spectral width of laser on bit error rate with different line codes. We created simulation with a variety of split ratios (from $1: 2$ to $1: 256$ and the higher attenuation value) and 2 higher values than were defined in E1 attenuation class (28 and $29.5 \mathrm{~dB}$ ). First of all, we designed a scenario for simulation of the maximum attenuation in ODN (Optical Distribution Networks) and we examined the values of bit error rate. Final attenuation was $35.5 \mathrm{~dB}$ for our simulation model. Miller's code has shown one of the best results for attenuation values. In particular, Miller's code had BER $10^{12}$ for $27.1 \mathrm{~dB}$, NRZ had $10^{7}$, and RZ had $10^{6}$. In comparison with other line codes, according to [1] only Miller's code has achieved the best results. All of these results were without forward error correction. FEC should also be implemented in future research.

\section{REFERENCES}

[1] Recommendation ITU-T G. 989. 1, 40-Gigabit-capable passive optical networks (NG-PON2): General requirements 2013.

[2] Recommendation ITU-T G. 989. 2, 40-Gigabit-capable passive optical networks 2 (NG-PON2): Physical media dependent (PMD) layer specification. 2013

[3] J. Lewin. Decoder for Delay-Modulation Coded Data, in IEEE Transactions on Communications, vol. 19, no. 5 pp. 719-723, 1971.

[4] P. Singh, D. K. Tripathi, and H. K. Dixit, Investigation of XOR operation in all-optical system with NRZ, RZ and Manchester modulation formats, in 2012 International Conference on Communications, Devices and Intelligent Systems (CODIS), pp. 417-420, 2012. 
[5] V. Tejkal, M. Filka, J. Sporik, P. Reichert, and P. Munster, The influence of binary modulations in passive optical network based on WDM, in Proc. 34th International Conference on Telecommunications and Signal Processing (TSP), Budapest, pp. 141-144, 2011.

[6] S. Yao, S. Fu, H. Wang, M. Tang, P. Shum, and D. Liu, Performance Comparison for NRZ, RZ, and CSRZ Modulation Formats in RS-DBS Nyquist WDM System, in Journal of Optical Communications and Networking, vol. 6, no. 4, p. 355, 2014.

[7] G. Bosco, A. Carena, V. Curri, R. Gaudino, and P. Poggiolini, On the use of NRZ, RZ, and CSRZ modulation at $40 \mathrm{~Gb} / \mathrm{s}$ with narrow DWDM channel spacing, in Journal of Lightwave Technology, vol. 20, no. 9, pp. 1694-1704, 2002.

[8] P. Munster, R. Sifta, T. Horvath, V. Novotny, and M. Filka. Polarization mode dispersion in NG- PON, in In Fourth Forum of Young Researchers. In the framework of International Forum Education Quality. Izhevsk, Russia: Publishing House, ISBN: 978-5-7526-0649-6, pp. 364-367, 2014.

[9] J. Mullerova, D. Korcek, and M. Dado, On wavelength blocking for XGPON coexistence with GPON and WDM-PON networks, in Proc. 14th International Conference on Transparent Optical Networks (ICTON), Coventry, pp. 1-4, 2012.

[10] A. M. Cailean, B. Cagneau, L. Chassagne, M. Dimian, and V. Popa Miller code usage in Visible Light Communications under the PHY I layer of the IEEE 802. 15. 7 standard, in Proc. 10th International Conference on Communications (COMM), Bucharest, pp. 1-4, 2014.

[11] J. Yu, Z. Jia, P. N. Ji, and T. Wang, $40 \mathrm{~Gb} / \mathrm{s}$ WDM Passive Optical Network with Centralized Lightwave Source, in Proc. OFC/NFOEC 2008 - 2008 Conference on Optical Fiber Communication/National Fiber Optic Engineers Conference, San Diego, CA, pp. 1-3, 2008.

[12] B. Liu, L. Zhang, X. Xin, and J. Yu, Constellation-masked secure communication technique for OFDM-PON, in Optics Express, vol. 20, no. 22, pp. 25161, 2012.

[13] Y. Luo, X. Zhou, F. Effenberger, X. Yan, G. Peng, Y. Qian, and Y. Ma Time and Wavelength-Division Multiplexed Passive Optical Network (TWDM-PON) for Next-Generation PON Stage 2 (NG-PON2), in Journal of Lightwave Technology, vol. 31, no. 4, pp. 587-593, 2013.

[14] Y. Kodama, The Whitham Equations for Optical Communications: Mathematical Theory of NRZ, in SIAM Journal on Applied Mathematics, vol. 59, no. 6, pp. 2162-2192, 1999.

Tomas Horvath was born in Havirov, Czech Republic on March 7, 1989. He received his M.Sc.degrees in Telecommunications from the Brno University of Technology, Brno, in 2013. His research interests include passive optical networks (xPON), optoelectronics and BitTorrent protocol. Currently, he has been actually post graduate student at Brno University of Technology, Department of Telecommunications and his topic of dissertation thesis is Optimization services in FTTx optical access networks. Technicka 12 - FEEC, BUT, Czech Republic 60190 or horvath@feec.vutbr.cz.

Radek Fujdiak was born in Czech Republic 1987. He received Bachelor (Teleinformatics) and Master degree (Telecommunication and Information Technique) in the Brno University of Technology (BUT). Now he is working on his Doctor Degree in Teleinformatics from the same University (BUT) His research interest include the mathematics, cryptology, smart grid and sensor networks. Technicka 12 - FEEC, BUT, Czech Republic 60190 or xfujdi00@phd.feec.vutbr.cz.

Milan Cucka Cucka was born in Vyskov (Czech Republic) 1989. He received his Bachelor Degree in Teleinformatics in 2012 and his master degree in Telecommunication and Information Technique in 2014. This titles received at Brno University of Technology. Now he is studiing his doctoral degree in the same university. His research is focused on optical fibers, mainly optical distributed systems which used this fibers, mainly optical distributed systems which used this fibers. Technicka 12 - FEEC, BUT, Czech Republic 60190 orxcucka00@phd.feec.vutbr.cz.
Marie Dankova graduated from the Mathematical engineering, Faculty of Mechanical Engineering, Brno University of Technology in 2014. She is currently working towards her PhD in Teleinformatics at Faculty of Electrical Engineering and Communication, Brno University of Technology. Technicka 12 - FEEC, BUT, Czech Republic 60190 or xdanko05@phd.feec.vutbr.cz.

Jiri Misurec received Master degree (Radioelectronics), CSc degree (Radioelectronics) and Doc. degree (Teleinformatics) in the Brno University of Technology (BUT). Now he is working as Department Leader in Department of Telecommunications BUT. His research interest include the signals, PLC, telecommunications, smart grid, power grid networks, networking and sensor networks. Antoninska 548/1 - BUT, Czech Republic 60190 or misurec@feec.vutbr.cz. 